\title{
Taylor-Couette instability in sphere suspensions
}

\author{
J. J. J. Gillissen* and H. J. Wilson \\ Department of Mathematics, University College London, Gower Street, London, WC1E 6BT, United Kingdom
}

(Received 19 December 2018; published 25 April 2019)

\begin{abstract}
We employ a rheological theory to show that circular Taylor-Couette flow of a suspension of non-Brownian spheres is less stable than that of a Newtonian fluid, at equal effective viscosity. The destabilization is related to the preferred orientation of the separation vector of the closely interacting spheres, in the compressive direction of the base flow. The results agree qualitatively with experimental observations from the literature.
\end{abstract}

DOI: 10.1103/PhysRevFluids.4.043301

\section{INTRODUCTION}

Taylor-Couette flow (TCF) is the flow between two concentric cylinders. When the outer cylinder is fixed, and when the rotation speed of the inner cylinder exceeds a critical value, the flow undergoes a centrifugal instability and develops an array of axisymmetric vortices [1]. A further increase of the rotation speed induces bifurcations into more complicated (wavy, oscillatory, etc.) vortices, and eventually into a state of fully developed turbulence. Owing to the connection with turbulence, and owing to the tractability by linear stability analysis, TCF is the subject of a vast amount of literature [2]. There is particular interest in TCF instability for non-Newtonian fluids. For instance, predicting experimentally measured onset conditions for instability provides a stringent test in the development of constitutive equations for complex fluids; see, e.g., Ref. [3].

In this work we study TCF of a suspension of non-Brownian spheres, which has experimentally been shown to be less stable than a Newtonian fluid, with equal effective viscosity [4]. These authors use a water-glycerol mixture, suspending density-matched polymethyl methacrylate spheres, with a radius of $a=115 \mu \mathrm{m}$, in the sphere volume fraction rage $0 \leqslant \phi \leqslant 0.3$ and in a flow cell, with an inner radius of $R_{1}=100.3 \mathrm{~mm}$, and an outer radius of $R_{2}=114.3 \mathrm{~mm}$, i.e., a radius ratio of $R_{2} / R_{1}=1.14$. The sphere Reynolds number is $\dot{\gamma} a^{2} / v \sim 10^{-2}$, where $v$ is the suspending fluid kinematic viscosity, $\dot{\gamma}=U / \Delta R$ is the shear rate, $U=\Omega R_{1}$ is the velocity of the inner cylinder, and $\Omega$ is the rotation speed of the inner cylinder. The experimental results are plotted as a function of $\phi$ in Fig. 1 with the markers, where the onset of instability is expressed by the effective, critical Taylor number:

$$
\mathrm{Ta}=\frac{U \Delta R}{v_{\text {eff }}} \sqrt{\frac{\Delta R}{R_{1}}} .
$$

Here $\Delta R=R_{2}-R_{1}$ is the gap width between the cylinders, and $v_{\text {eff }}(\phi)$ is the concentrationdependent and shear rate-invariant, effective suspension viscosity, which was measured in Ref. [4], and which was parameterized by

$$
\frac{v_{\text {eff }}}{v}=\left(1-\frac{\phi}{0.55}\right)^{-1.83} .
$$

The reduction in the critical, effective Taylor number, observed in Fig. 1, indicates that sphere suspensions are less stable than Newtonian liquids, with equivalent effective viscosities. At small

*jurriaangillissen@gmail.com 


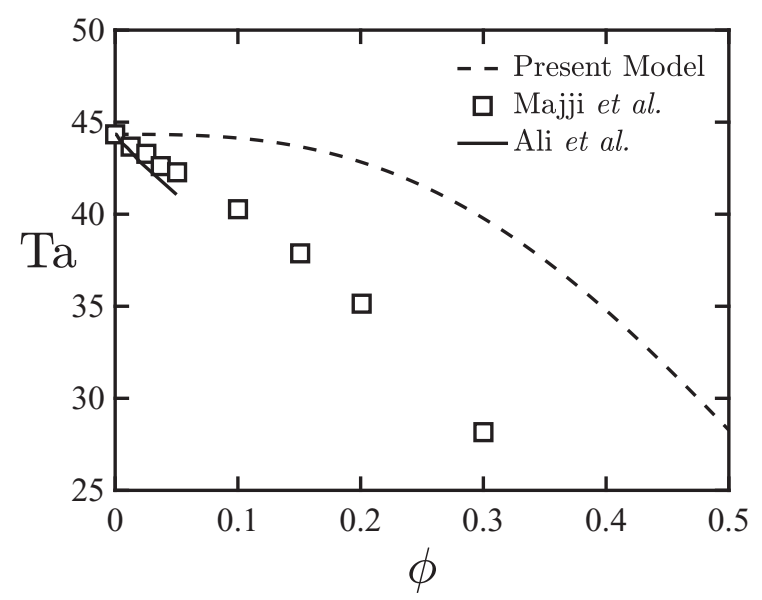

FIG. 1. The critical, effective Taylor number Ta [Eq. (1)] as a function of the sphere volume fraction $\phi$. Comparison between the experimental data of Ref. [4] (markers), the two-fluid theory of Ref. [5] (solid line), and the present rheological theory (dashed line).

sphere concentrations $\phi$, this effect is believed to be related to the slip between the solid and the fluid phase and the associated, inhomogeneous spatial sphere distribution [6].

At small $\phi$, the destabilization of TCF due to non-Brownian spheres has been captured by linear stability analysis of axisymmetric perturbations of the two fluid theory, using a radius ratio of $R_{2} / R_{1}=1.18$ [5]. Under the assumptions of $\phi \ll 1$, and a small sphere Reynolds number $\dot{\gamma} a^{2} / v \ll 1$, these authors adopt the following momentum equations for the liquid and solid phases:

$$
\begin{aligned}
& \partial_{t} \boldsymbol{u}+\boldsymbol{u} \cdot \nabla \boldsymbol{u}=-\nabla p+v \nabla^{2} \boldsymbol{u}+\phi \tau^{-1}(\boldsymbol{v}-\boldsymbol{u}), \\
& \partial_{t} \boldsymbol{v}+\boldsymbol{v} \cdot \nabla \boldsymbol{v}=-\nabla p+\tau^{-1}(\boldsymbol{u}-\boldsymbol{v})
\end{aligned}
$$

Here $\boldsymbol{u}$ is the fluid velocity, $\boldsymbol{v}$ is the (locally) averaged sphere velocity, $p$ is the fluid pressure, and $\tau=2 a^{2} /(9 v)$ is the sphere relaxation time. In steady shear flow, the effective viscosity of the two fluid theory [Eq. (3)] equals $v_{\text {eff }}=v$. Under time-varying conditions, the magnitude of the interfacial drag force can be estimated, by ignoring the pressure term in Eq. (3b), and by assuming, that $\boldsymbol{u}=\hat{\boldsymbol{u}} \cos (\omega t)$, with $\hat{\boldsymbol{u}}$ and $\omega$ constants, such that the sphere velocity is governed by $\partial_{t} \boldsymbol{v}=$ $\tau^{-1}[\hat{\boldsymbol{u}} \cos (\omega t)-\boldsymbol{v}]$, which, under the additional assumption, that $\omega \tau \ll 1$, is solved by

$$
\phi \tau^{-1}(\boldsymbol{u}-\boldsymbol{v})=-\phi \partial_{t} \boldsymbol{u} .
$$

Equation (4) illustrates, that, in the limit of $\omega \tau \ll 1$, the interfacial drag force is independent of the sphere size, and $\phi$ is the only nondimensional parameter that is introduced by the addition of the spheres.

The results of the linear stability analysis of Eq. (3) in TCF are plotted in Fig. 1 by the solid line [5]. These results agree well with the experimental results of Ref. [4] (markers). The theory [Eq. (3)] ignores interactions between spheres, and the computations of Ref. [5] are therefore restricted to $0<\phi<0.05$. For larger $\phi$, sphere interactions become important, and these effects are not captured by Eq. (3). These interactions give rise to an additional stress, which depends on the relative arrangement of the spheres, referred to as the microstructure; see, e.g., Refs. [7,8]. In this regard, it is known that suspensions behave (generalized) Newtonian for $\phi \lesssim 0.2$, while for $\phi \gtrsim 0.2$, the anisotropic microstructure gives rise to deviations from Newtonian behavior, e.g., substantial normal stress differences in shear flow. In these dense systems, the dominant effect of the spheres is the extra stress, while the interfacial drag force, captured by Eq. (3), is expected to play an inferior role. In this work we use our previously developed sphere stress theory, to investigate 


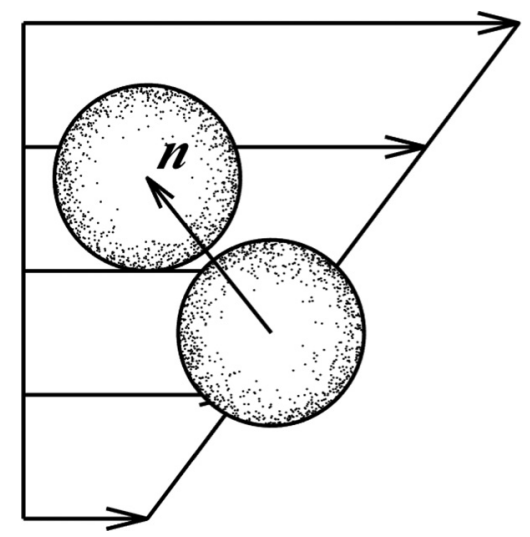

FIG. 2. The sphere pair separation unit vector $\boldsymbol{n}$.

whether the additional, non-Newtonian stress has either a stabilizing or a destabilizing effect on the circular TCF.

\section{SUSPENSION STRESS THEORY}

The suspension stress theory ignores all nonhydrodynamic forces and assumes that the extra stress is induced by the lubrication forces between the spheres [7]. This assumption is valid for a negligible sphere Reynolds number $\dot{\gamma} a^{2} / \nu \ll 1$ and for intermediate volume fractions $0.2<\phi<$ 0.5 , while for lower volume fractions the lubrication approximation fails, and for higher volume fractions direct contacts between the spheres become important [8]. The hydrodynamic stress, induced by the spheres, is modeled as [7]

$$
\sigma=2 v \alpha s:\langle\boldsymbol{n n n n}\rangle,
$$

where $s=\frac{1}{2}\left(\nabla \boldsymbol{u}+\nabla \boldsymbol{u}^{T}\right)$ is the rate of strain tensor, $\nabla \boldsymbol{u}$ is the velocity gradient tensor, $\boldsymbol{u}$ is the velocity vector, $\alpha$ is the stress parameter, which depends on $\phi$, and is further specified in Eq. (11a), and $\langle\boldsymbol{n n n n}\rangle=\int \Psi(\boldsymbol{n}) \boldsymbol{n n n n n} d^{2} \boldsymbol{n}$, is the fourth-order moment of the distribution $\Psi$ of the sphere pair separation unit vector $\boldsymbol{n}$ (Fig. 2). In Eq. (5) there is no explicit dependence of $\boldsymbol{\sigma}$ on the sphere radius $a$, since, in Stokesian systems, there are no variables to nondimensionalize $a$ with.

Under the assumption that $\Psi$ is weakly anisotropic, $\langle\boldsymbol{n n n n n}\rangle$ can be expressed as a linear function of the second-order moment $\boldsymbol{a}=\langle\boldsymbol{n n}\rangle=\int \Psi(\boldsymbol{n}) \boldsymbol{n} \boldsymbol{n} \boldsymbol{d}^{2} \boldsymbol{n}$ as follows [9]:

$$
\begin{aligned}
\left\langle n_{i} n_{j} n_{k} n_{l}\right\rangle= & -\frac{1}{35}\left\langle n_{m} n_{m}\right\rangle\left(\delta_{i j} \delta_{k l}+\delta_{i k} \delta_{j l}+\delta_{i l} \delta_{j k}\right) \\
& +\frac{1}{7}\left(\delta_{i j}\left\langle n_{k} n_{l}\right\rangle+\delta_{i k}\left\langle n_{j} n_{l}\right\rangle+\delta_{i l}\left\langle n_{j} n_{k}\right\rangle+\left\langle n_{i} n_{j}\right\rangle \delta_{k l}+\left\langle n_{i} n_{k}\right\rangle \delta_{j l}+\left\langle n_{i} n_{l}\right\rangle \delta_{j k}\right),
\end{aligned}
$$

such that the nonisotropic part of the sphere stress [Eq. (5)] reads

$$
\frac{\sigma}{2 v \alpha}=-\frac{2}{35} s+\frac{2}{7}(s \cdot a+a \cdot s) .
$$

In $x y$-shear flow with shear rate $\dot{\gamma}$ :

$$
s=\frac{\dot{\gamma}}{2}\left(\begin{array}{lll}
0 & 1 & 0 \\
1 & 0 & 0 \\
0 & 0 & 0
\end{array}\right),
$$


the distribution $\Psi$ is assumed to be a superposition of the isotropic distribution and a distribution that has a preferred alignment in the compressive axis $\boldsymbol{n}^{c}=(-1,1,0) / \sqrt{2}[10]$ :

$$
\boldsymbol{a}=\frac{1-\beta}{3} \boldsymbol{\delta}+\beta \boldsymbol{n}^{c} \boldsymbol{n}^{c}=\left(\begin{array}{ccc}
\frac{1}{3}+\frac{\beta}{6} & -\frac{\beta}{2} & 0 \\
-\frac{\beta}{2} & \frac{1}{3}+\frac{\beta}{6} & 0 \\
0 & 0 & \frac{1}{3}-\frac{\beta}{3}
\end{array}\right),
$$

where $\beta$ is referred to as the "anisotropy parameter." Combining Eqs. (6)-(8) gives the following stress in the $x y$-shear flow:

$$
\frac{\sigma}{2 \nu \gamma \alpha}=\left(\begin{array}{ccc}
-\frac{\beta}{7} & \frac{(5 \beta+7)}{105} & 0 \\
\frac{(5 \beta+7)}{105} & -\frac{\beta}{7} & 0 \\
0 & 0 & 0
\end{array}\right),
$$

which reduces to a Newtonian stress for $\beta=0$, and which corresponds to the following effective viscosity:

$$
\frac{v_{\text {eff }}}{v}-1=\frac{\sigma_{12}}{v \dot{\gamma}}=\alpha \frac{2(5 \beta+7)}{105},
$$

and the following, relative, second normal stress difference:

$$
\zeta_{2}=\frac{\sigma_{22}-\sigma_{33}}{\sigma_{12}}=-\frac{15 \beta}{5 \beta+7}
$$

and the first normal stress difference is zero by construction $\zeta_{1}=\left(\sigma_{11}-\sigma_{22}\right) / \sigma_{12}=0$, in qualitative agreement with the experimental literature. In this work we tune $\alpha$ and $\beta$ as

$$
\alpha=\frac{5\left(3-2 \phi^{2}\right)}{2}\left[\left(1-\frac{\phi}{0.55}\right)^{-1.83}-1\right]
$$

and

$$
\beta=\frac{14 \phi^{2}}{5\left(3-2 \phi^{2}\right)},
$$

such that Eq. (10) reduces to the empirical effective suspension viscosity $v_{\text {eff }} / v$ [Eq. (2)], and to the empirical, relative, second normal stress difference:

$$
\zeta_{2}=-2 \phi^{2}
$$

It is emphasized, that with Eq. (11), the theory [Eqs. (6) and (8)] exactly reproduces the experimentally measured shear stress and second normal stress difference. It is also noted that contact forces result in a positive first normal stress difference [8]. Here we ignore these effects and restrict our focus to purely hydrodynamic systems, for which the non-Newtonian rheology in shear flow is dominated by the second normal stress difference, i.e., $\left|\zeta_{2}\right| \gg\left|\zeta_{1}\right|$, as is usually observed in shear rate-invariant sphere suspensions [11-14].

\section{STABILITY ANALYSIS}

TCF is described in cylindrical coordinates, $r, \theta, z=1,2,3$, and is governed by the continuity equation,

$$
\nabla \cdot \boldsymbol{u}=0
$$

and by the momentum equation,

$$
\partial_{t} \boldsymbol{u}=\nabla \cdot\left[-\boldsymbol{u} \boldsymbol{u}-p \boldsymbol{\delta}+v\left(\nabla \boldsymbol{u}+\nabla \boldsymbol{u}^{T}\right)+\boldsymbol{\sigma}\right] .
$$


The velocity gradient tensor in cylindrical coordinates reads

$$
\nabla_{i} u_{j}=\left(\begin{array}{ccc}
\partial_{r} u_{r} & \partial_{r} u_{\theta} & \partial_{r} u_{z} \\
-r^{-1} u_{\theta} & r^{-1} u_{r} & 0 \\
\partial_{z} u_{r} & \partial_{z} u_{\theta} & \partial_{z} u_{z}
\end{array}\right) .
$$

We compute the linear stability of the laminar solution to Eqs. (6), (8), (13), and (14), w.r.t. axisymmetric perturbations, that are harmonic functions of $z$, with a wave number $k$. Although nonaxisymmetric modes are observed in the experiments of Ref. [4], we restrict focus to axisymmetric modes, which serves the purpose of elucidating the basic destabilizing effect of the sphere stress, which is related to the anisotropic microstructure. We decompose the hydrodynamic fields, e.g., the fluid velocity: $\boldsymbol{u}=\boldsymbol{U}(r)+\boldsymbol{u}^{\prime}(r, t) \exp (i k z)$, into a base state, denoted by a capital letter: $\boldsymbol{U}$, and a perturbation, denoted by a prime: $\boldsymbol{u}^{\prime} \exp (i k z)$.

Since the effective viscosity $v_{\text {eff }}$ [Eq. (2)] is shear rate invariant and therefore position invariant, the base velocity field $\boldsymbol{U}=U_{\theta} \boldsymbol{e}_{\theta}$ is similar to that of a Newtonian fluid, and is governed by the azimuthal component of the momentum equation [Eq. (14)]: $\left(\partial_{r}+2 r^{-1}\right)\left(\partial_{r}-2 r^{-1}\right) U_{\theta}=0$, which gives

$$
U_{\theta}=\frac{\Omega r}{1-R_{2}^{2} / R_{1}^{2}}+\frac{R_{1}^{2} \Omega / r}{1-R_{1}^{2} / R_{2}^{2}} .
$$

The perturbations are governed by the continuity equation [Eq. (13)]:

$$
\left(\partial_{r}+r^{-1}\right) u_{r}^{\prime}+i k u_{z}^{\prime}=0
$$

and by the three components of the linearized momentum equation [Eq. (14)]:

$$
\begin{gathered}
\partial_{t} u_{r}^{\prime}=-\partial_{r} p^{\prime}+v\left(\partial_{r}^{2}+r^{-1} \partial_{r}-r^{-2}-k^{2}\right) u_{r}^{\prime}+2 r^{-1} U_{\theta} u_{\theta}^{\prime}+\left(\partial_{r}+r^{-1}\right) \sigma_{r r}^{\prime}+i k \sigma_{z r}^{\prime}-r^{-1} \sigma_{\theta \theta}^{\prime}, \\
\partial_{t} u_{\theta}^{\prime}=v\left(\partial_{r}^{2}+r^{-1} \partial_{r}-r^{-2}-k^{2}\right) u_{\theta}^{\prime}-\left[\left(\partial_{r}+r^{-1}\right) U_{\theta}\right] u_{r}^{\prime}+\left(\partial_{r}+2 r^{-1}\right) \sigma_{r \theta}^{\prime}+i k \sigma_{z \theta}^{\prime},
\end{gathered}
$$

and

$$
\partial_{t} u_{z}^{\prime}=-i k p^{\prime}+v\left(\partial_{r}^{2}+r^{-1} \partial_{r}-k^{2}\right) u_{z}^{\prime}+\left(\partial_{r}+r^{-1}\right) \sigma_{r z}^{\prime}+i k \sigma_{z z}^{\prime} .
$$

Here the perturbed stress [Eq. (6)] reads

$$
\frac{\sigma^{\prime}}{2 v \alpha}=-\frac{2}{35} s^{\prime}+\frac{2}{7}\left(s^{\prime} \cdot a+a \cdot s^{\prime}\right),
$$

where $s=\frac{1}{2}\left(\nabla \boldsymbol{u}+\nabla \boldsymbol{u}^{T}\right)$ is the rate of strain tensor, the cylindrical components of the velocity gradient $\boldsymbol{\nabla} \boldsymbol{u}$ are given in Eq. (15), and the cylindrical components of the conformation tensor $\boldsymbol{a}$ are identical to its Cartesian components, which are given in Eq. (8). We numerically solve the $r$-dependent velocity perturbations $\boldsymbol{u}^{\prime}$ using Chebyshev discretization on 30 collocation points [15]. We fix the Couette cell radius ratio to $R_{2} / R_{1}=1.14$, similarly as in the experimental study of Ref. [4].

The velocity perturbation evolution equations [Eqs. (16-21)] including the boundary conditions $\left(\boldsymbol{u}^{\prime}=\mathbf{0}\right.$ on the walls) are written in matrix form:

$$
\boldsymbol{M}_{1} \cdot \partial_{t} \boldsymbol{q}^{\prime}=\boldsymbol{M}_{2} \cdot \boldsymbol{q}^{\prime}
$$

and the growth rates are found by solving the corresponding generalized eigenvalue problem in FORTRAN using the ZGGEV routine from the LAPACK library. For all cases discussed below, the eigenvalue of the most unstable axisymmetric mode is real-valued, i.e., nonoscillatory.

The rotation rate $\Omega$ is varied to find the onset of instability, which corresponds to a sign change of this largest eigenvalue. The onset point is expressed by the effective, critical Taylor number Ta [Eq. (1)]. 


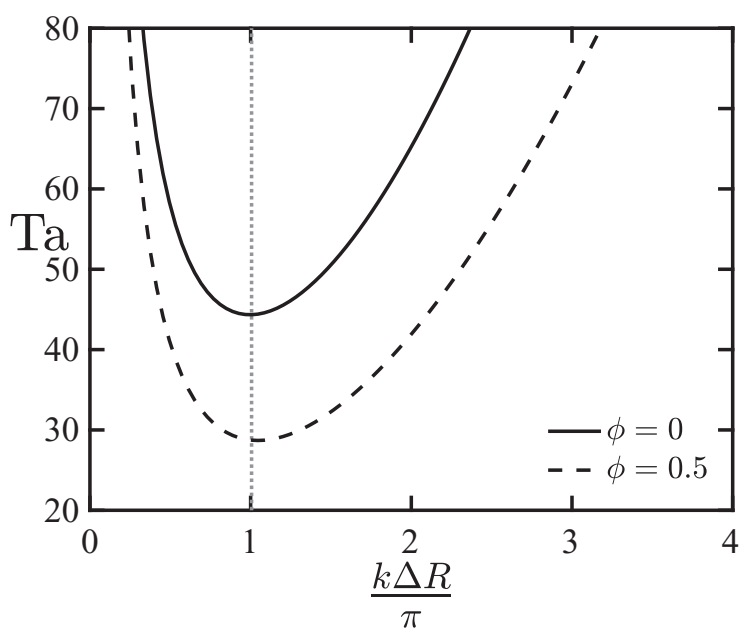

FIG. 3. The computed, critical, effective Taylor number Ta [Eq. (1)] as a function of the normalized, spanwise wave number $k \Delta R / \pi$, for the Newtonian fluid (sphere volume fraction $\phi=0$; solid line) and for a suspension with $\phi=0.5$ (dashed line). The vertical, dotted line coincides with the minima of the curves.

\section{RESULTS}

Figure 3 shows the computed Ta as a function of the spanwise wave number $k \Delta R / \pi$ for the Newtonian fluid (sphere volume fraction $\phi=0$ ) and for a suspension with $\phi=0.5$. It is seen that for both cases the minimum, critical, effective Taylor number occurs at $k \Delta R / \pi \approx 1$. It is furthermore seen that the spheres are destabilizing, i.e., for $\phi=0.5$, the predicted, critical, effective Taylor number is reduced by roughly $40 \%$.

Figure 4 shows the velocity components of the most unstable mode, in the Newtonian fluid $(\phi=0)$ at the critical Taylor number of $\mathrm{Ta} \approx 44$, and in a suspension with $\phi=0.5$, at the critical, effective Taylor number of Ta $\approx 28$. Note that these modes are normalized, and their magnitude has no physical significance. In both cases, the modes are similar and are dominated by the azimuthal velocity component. The ratio of the cross-stream velocity fluctuations to the azimuthal velocity fluctuations is slightly larger in the suspension than in the Newtonian fluid.

Figure 1 shows the computed, critical, effective Taylor number for the suspension (dashed line) as a function of the sphere volume fraction $\phi$. The figure shows that, when compared at equal $v_{\text {eff }} / \nu$, the spheres have a destabilizing effect for $\phi \gtrsim 0.2$, and a negligible effect for $\phi \lesssim 0.2$. This concentration dependence corresponds to the non-Newtonian nature of the suspension, which is characterized by the second normal stress difference $\zeta_{2}$ [Eq. (12)], which is non-negligible, only for $\phi \gtrsim 0.2$.

The destabilization can be understood by the alignment of the microstructure with the base deformation [Eq. (8)]. To this end, we introduce an alternative definition for the effective "base flow viscosity" $v_{\text {eff }}$, as the ratio of the dissipation of the base kinetic energy, due to the total (sphere plus solvent) stress and due to the solvent stress:

$$
\frac{v_{\text {eff }}}{v}-1=\frac{S: \Sigma}{2 v S: S}
$$

By inserting for $S$ the expression for the base deformation rate [Eq. (7)] and for $\boldsymbol{\Sigma}$ the expression for the base sphere stress [Eq. (9)], Eq. (23) reduces to Eq. (10a). Similarly, we introduce the effective "vortex viscosity" $v^{\prime}$, which is based on the dissipation of the perturbed kinetic energy:

$$
\frac{v^{\prime}}{v}-1=\frac{\boldsymbol{s}^{\prime}: \boldsymbol{\sigma}^{\prime}}{2 v \mathbf{s}^{\prime}: \mathbf{s}^{\prime}}
$$



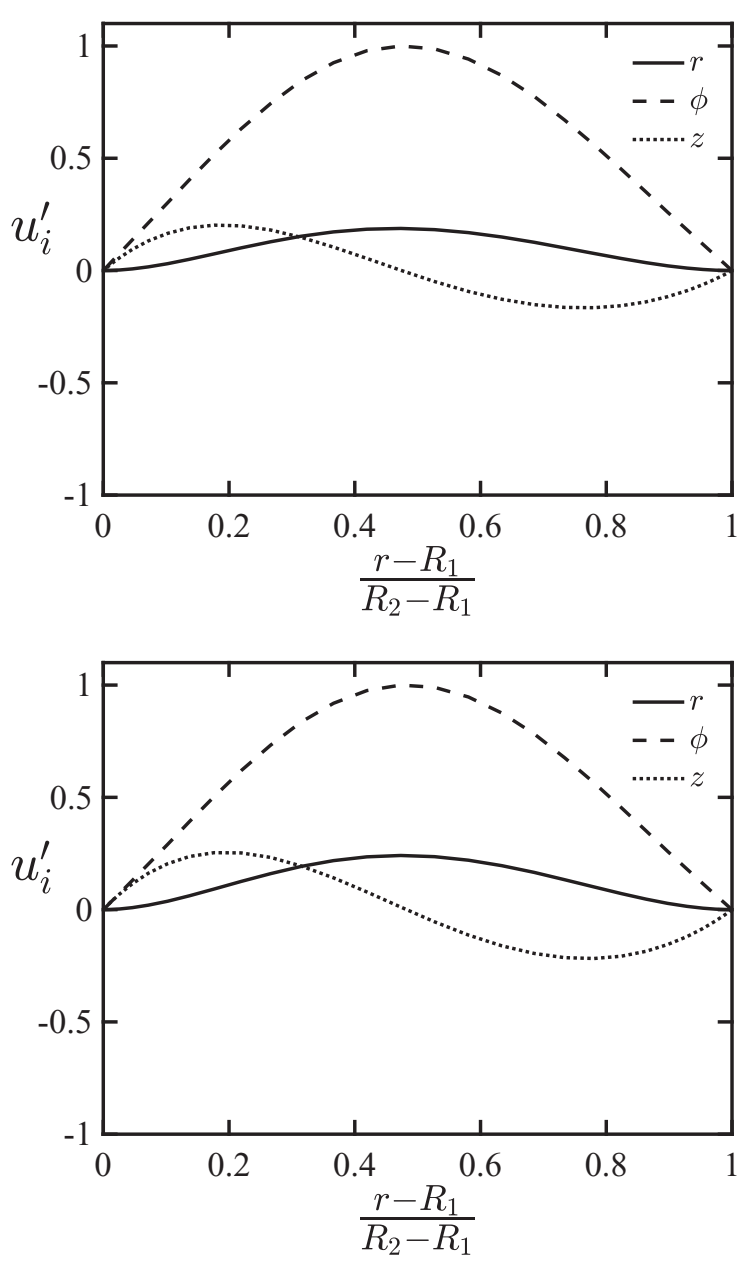

FIG. 4. (top) Most unstable eigenmode in the Newtonian fluid, i.e., using a sphere volume fraction of $\phi=0$, and a Taylor number [Eq. (1)] at the critical value of Ta $\approx 44$. (bottom) Most unstable axisymmetric eigenmode in a sphere suspension, using a sphere volume fraction of $\phi=0.5$, and an effective Taylor number [Eq. (1)] at the critical value of $\mathrm{Ta} \approx 28$.

Inserting the expressions for the perturbed stress $\sigma^{\prime}$ [Eqs. (8) and (21)] into Eq. (24) gives

$$
\frac{v^{\prime}}{v}-1=\alpha\left[\frac{2}{15}+\frac{4 \beta}{7}\left(-\frac{1}{3}+\xi\right)\right],
$$

where the alignment factor,

$$
\xi=\frac{\boldsymbol{s}^{\prime}:\left(\boldsymbol{s}^{\prime} \cdot \boldsymbol{n}^{c} \boldsymbol{n}^{c}\right)}{\boldsymbol{s}^{\prime}: \boldsymbol{s}^{\prime}},
$$

measures the deformation of the secondary flow in the compressive direction of the base flow. For perfectly aligned biaxial deformation, we see that $\xi=\frac{1}{2}$ and $v^{\prime}=v_{\text {eff }}$. Since the secondary flow deformation is not perfectly aligned with the base flow deformation, we see that $\xi<\frac{1}{2}$. This means that $v_{\text {eff }}>v^{\prime}$, i.e., the spheres impose more friction to the base flow than to the secondary vortices. Since the instability involves the dynamics of the vortices, we may model the onset by $\mathrm{Ta}^{\prime} \approx 44$, where $\mathrm{Ta}^{\prime}$ is the critical Taylor number, that is based on $v^{\prime}$, i.e., $\mathrm{Ta}^{\prime}=\left(U \Delta R / v^{\prime}\right) \sqrt{\Delta R / R_{1}}$. 
Since $v_{\text {eff }}>v^{\prime}$, this corresponds to a critical Taylor number based on $v_{\text {eff }}$, that is smaller than the Newtonian value, i.e., $\mathrm{Ta}=\left(U \Delta R / v_{\text {eff }}\right) \sqrt{\Delta R / R_{1}}<44$, which explains the destabilizing effect.

The theoretical results in Fig. 1 (dashed line) show less destabilization, as compared to the experiments in Ref. [4] (markers). As discussed above, the discrepancy may be attributed to the neglect of sphere inertia in the present theory. Another possible cause for the discrepancy is the assumption of axisymmetry of the instability modes. For small volume fractions $\phi<0.05$, this assumption agrees with the experiments of Ref. [4], and in this regime the discrepancy is therefore most likely due to neglecting inertia. The importance of sphere inertia is supported by the agreement between the axisymmetric two-fluid theory [Eq. (3)] of Ref. [5] and the experimental data for $\phi<0.05$ (see Fig. 1). At larger $\phi$, the two-fluid theory [Eq. (3)] does not hold, and to better capture this regime, we propose for future work a nonaxisymmetric stability analysis, which includes both sphere inertia and sphere stress, by combining the two fluid theory [Eq. (3)], with our sphere stress theory [Eqs. (6) and (8)].

\section{v. CONCLUSION}

We have theoretically predicted a destabilization of the circular Taylor-Couette flow w.r.t. axisymmetric perturbations, due to the presence of non-Brownian spheres. The non-Newtonian character of the suspension base flow, is characterized by the second normal stress difference, while the first normal stress difference is assumed zero. The destabilization can be understood by the alignment between the microstructure and the base deformation.

\section{ACKNOWLEDGMENT}

We would like to acknowledge financial support from the Engineering and Physical Sciences Research Council of the United Kingdom Grant No. EP/N024915/1.

[1] G. I. Taylor, Stability of a viscous liquid contained between two rotating cylinders, Phil. Trans. R. Soc. Lond. Ser. A 223, 289 (1923).

[2] M. A. Fardin, C. Perge, and N. Taberlet, The hydrogen atom of fluid dynamics-Introduction to the Taylor-Couette flow for soft matter scientists, Soft Matter 10, 3523 (2014).

[3] J. J. J. Gillissen and H. J. Wilson, Taylor Couette instability in disk suspensions, Phys. Rev. Fluids 3, 113903 (2018).

[4] M. V. Majji, S. Banerjee, and J. F. Morris, Inertial flow transitions of a suspension in Taylor-Couette geometry, J. Fluid Mech. 835, 936 (2018).

[5] M. E. Ali, D. Mitra, J. A Schwille, and R. M. Lueptow, Hydrodynamic stability of a suspension in cylindrical Couette flow, Phys. Fluids 14, 1236 (2002).

[6] M. V. Majji and J. F. Morris, Inertial migration of particles in Taylor-Couette flows, Phys. Fluids 30, 033303 (2018).

[7] J. J. J. Gillissen and H. J. Wilson, Modeling sphere suspension microstructure and stress, Phys. Rev. E 98, 033119 (2018).

[8] J. J. J. Gillissen and H. J. Wilson, The effect of normal contact forces on the stress in shear rate invariant particle suspensions, Phys. Rev. Fluids 4, 013301 (2019).

[9] E. J. Hinch and L. G. Leal, Constitutive equations in suspension mechanics. Part 2. Approximate forms for a suspension of rigid particles affected by Brownian rotations, J. Fluid Mech. 76, 187 (1976).

[10] F. Blanc, E. Lemaire, A. Meunier, and F. Peters, Microstructure in sheared non-Brownian concentrated suspensions, J. Rheol. 57, 273 (2013).

[11] A. Singh and P. R. Nott, Experimental measurements of the normal stresses in sheared Stokesian suspensions, J. Fluid Mech. 490, 293 (2003). 
[12] I. E. Zarraga, D. A. Hill, and D. T. Leighton Jr, The characterization of the total stress of concentrated suspensions of noncolloidal spheres in Newtonian fluids, J. Rheol. 44, 185 (2000).

[13] É. Couturier, F. Boyer, O. Pouliquen, and É. Guazzelli, Suspensions in a tilted trough: Second normal stress difference, J. Fluid Mech. 686, 26 (2011).

[14] S.-C. Dai, E. Bertevas, F. Qi, and R. I. Tanner, Viscometric functions for noncolloidal sphere suspensions with Newtonian matrices, J. Rheol. 57, 493 (2013).

[15] C. Canuto, M. Y. Hussaini, A. Quarteroni, and A. Thomas Jr., Spectral Methods in Fluid Dynamics (Springer, Berlin, 2012). 